\title{
Is human blood a good surrogate for brain tissue in transcriptional studies?
}

\author{
Chaochao Cai ${ }^{1,2}$, Peter Langfelder ${ }^{1}$, Tova F Fuller ${ }^{1}$, Michael C Oldham³ ${ }^{3}$, Rui Luo ${ }^{1}$, Leonard H van den Berg ${ }^{4}$, \\ Roel A Ophoff ${ }^{4,5}$, Steve Horvath ${ }^{1,2^{*}}$
}

\begin{abstract}
Background: Since human brain tissue is often unavailable for transcriptional profiling studies, blood expression data is frequently used as a substitute. The underlying hypothesis in such studies is that genes expressed in brain tissue leave a transcriptional footprint in blood. We tested this hypothesis by relating three human brain expression data sets (from cortex, cerebellum and caudate nucleus) to two large human blood expression data sets (comprised of 1463 individuals).

Results: We found mean expression levels were weakly correlated between the brain and blood data ( $r$ range: $[0.24,0.32])$. Further, we tested whether co-expression relationships were preserved between the three brain regions and blood. Only a handful of brain co-expression modules showed strong evidence of preservation and these modules could be combined into a single large blood module. We also identified highly connected intramodular "hub" genes inside preserved modules. These preserved intramodular hub genes had the following properties: first, their expression levels tended to be significantly more heritable than those from non-preserved intramodular hub genes $\left(p<10^{-90}\right)$; second, they had highly significant positive correlations with the following cluster of differentiation genes: CD58, CD47, CD48, CD53 and CD164; third, a significant number of them were known to be involved in infection mechanisms, post-transcriptional and post-translational modification and other basic processes.

Conclusions: Overall, we find transcriptome organization is poorly preserved between brain and blood. However, the subset of preserved co-expression relationships characterized here may aid future efforts to identify blood biomarkers for neurological and neuropsychiatric diseases when brain tissue samples are unavailable.
\end{abstract}

\section{Background}

There is no clear consensus regarding the use of bloodbased gene expression data for addressing neurological and neuroscientific research questions. On the one hand, gene expression levels in whole blood are only weakly correlated with those in brain tissue [1,2]. On the other hand, blood gene expression profiles have been used to study neuropsychiatric diseases such as bipolar disorder and schizophrenia [3-6], as well as neurological diseases such as Amyotrophic Lateral Sclerosis [7], Huntington's disease [8], Alzheimer's disease [9], and chronic fatigue syndrome [10]. There are at least two major reasons why the relationship between human brain and human blood

\footnotetext{
* Correspondence: shorvath@mednet.ucla.edu

'Department of Human Genetics, David Geffen School of Medicine,

University of California Los Angeles, Los Angeles, CA 90095, USA

Full list of author information is available at the end of the article
}

expression profiles remains poorly understood. The first reason concerns data quality and quantity: it is notoriously difficult to measure human brain tissue expression levels because of potential biases from post-mortem effects and relatively low sample sizes. The second reason is that most previous studies have focused on the preservation of mean expression levels, as opposed to the preservation of co-expression relationships. Given that the human brain transcriptome is organized into biologically meaningful co-expression modules [11-14], it is important to study the preservation of this organization in blood.

Because human brain expression data is derived from post-mortem brain tissue, special attention must be paid to RNA quality, post-mortem interval, and $\mathrm{pH}$. To minimize the influence of these factors, we used highly reproducible and validated brain gene expression data
C Biomed Central

(c) 2010 Cai et al; licensee BioMed Central Ltd. This is an Open Access article distributed under the terms of the Creative Commons Attribution License (http://creativecommons.org/licenses/by/2.0), which permits unrestricted use, distribution, and reproduction in any medium, provided the original work is properly cited. 
sets from a recent meta-analysis of publicly available brain expression data [12]. Data set 1 (referred to as CTX) consisted of 67 control samples from 67 individuals representing four cortical areas [15-17]. Data set 2 (referred to as $\mathrm{CN}$ ) consisted of 27 control samples from 27 individuals taken from the head of the caudate nucleus [18]. Data set 3 (referred to as CB) consisted of 24 control samples from 24 individuals taken from cerebellar hemisphere [15].

By applying weighted gene co-expression network analysis (WGCNA) [19-21] to these data sets, Oldham et al. (2008) identified 19 cortex (CTX) modules, 23 caudate nucleus $(\mathrm{CN})$ modules, and 22 cerebellum $(\mathrm{CB})$ modules. These modules were defined as branches of a hierarchical clustering tree and were labeled by different colors. Many modules were highly preserved across the three brain regions, which was why they received the same color label. For example, $45 \%\left(p=2.8 \times 10^{-53}\right)$ of genes overlapped between the yellow cortex module (labeled yellow/CTX) and yellow caudate nucleus module (labeled yellow/CN). Similarly, 46\% $\left(p=1.1 \times 10^{-54}\right)$ of genes overlapped between the blue/CTX and the blue/CN modules [12]. By considering cell type-specific markers, several brain modules were found to contain genes that are preferentially expressed in oligodendrocytes, astrocytes or neurons [12].

Here we report the results of a comprehensive statistical analysis by cross-referencing the brain expression data with two large blood data sets (comprising a total of 1463 individuals). While previous studies have focused on the preservation of individual gene expression levels across the two tissues, we also investigated the preservation of gene co-expression modules. Since oligodenrocytes, astrocytes, and neurons are not present in blood, we were not surprised that only a handful of human brain modules showed evidence of preservation in human blood. Furthermore, we determined that these preserved modules could be combined into a single large module in blood. We also found that preserved intramodular hub genes tended to have heritable blood expression levels and were highly correlated with a small set of cluster of differentiation (CD) genes.

\section{Results}

\section{Blood gene expression data}

We used whole blood gene expression data from healthy controls in a Dutch data set $(\mathrm{n}=405)$ and published lymphocyte gene expression data $(n=1240)$, herein referred to as the San Antonio Family Heart Study (SAFHS) data set [22]. The Dutch data set originally consisted of 405 peripheral blood samples from healthy individuals $(50.4 \%$ men and $49.6 \%$ women, mean age 56.4 and range from 19-88). This data set was analyzed with Illumina Human HT-12 microarrays. The SAFHS data set originally consisted of 1240 lymphocytes samples obtained from 1240 individuals $(40.8 \%$ men and $59.2 \%$ women, mean age 39.3 and range from 15-94). This data set was analyzed with Illumina WG-6 microarrays. Using hierarchical clustering with inter-array correlations as a distance measure, we identified potential outlying arrays in an unbiased fashion. Since outlying arrays showed relatively low correlations with the other arrays (across the genes), they were deemed suspicious. To err on the side of caution, we removed these suspicious arrays from the analysis. Potential batch effects (due to different hybridization dates) were also removed using ComBat [23]. These are the same data pre-processing steps that Oldham et al. (2008) used in the brain data analysis. More sample pre-processing information can be found in Additional file 1.

After these pre-processing steps, 380 samples remained in the Dutch data set and 1084 samples remained in the SAFHS data set. Multiple probes corresponding to one gene (symbol) were combined into one measurement. Next, we merged the Affymetrix (brain) data with the Illumina (blood) data by gene symbol, which resulted in 8799 genes in each data set.

\section{Preservation of mean expression levels and connectivity}

We first studied the preservation of mean gene expression levels of the 8799 genes between brain and blood. The pairwise scatterplots in Additional file 2 related mean expression values in the three brain regions to mean expression values in the two blood data sets. We found significant but weak correlations ( $r$ range: $[0.24,0.32])$ between mean expression in brain and mean expression in blood.

Next we investigated the extent to which co-expression patterns were preserved between brain and blood. For each gene, the network connectivity (also known as degree) is defined as the sum of its connection strengths with all other genes in the network. Thus, connectivity measures how correlated a gene is with all other genes (see Methods). Genes with high connectivity are informally referred to as "hub" genes. Overall, we found that gene connectivity was even less preserved ( $r$ range: [0.021,0.11], Additional file 3) in blood than mean expression levels. These results show that global coexpression relationships are poorly preserved between brain and blood. However, Additional file 3 also shows some genes with high connectivity in both data sets. These genes may be part of sets of genes (co-expression modules) that are preserved between the two tissues. A more focused analysis that considered individual modules did reveal some evidence of preservation between the two tissues, as described below. 


\section{Preservation of brain co-expression modules in blood}

Oldham et al. (2008) applied rigorous gene filters to the brain data set to ensure that transcripts were present and had high connectivity in the brain data (see the Supplemental Information of Oldham et al. 2008). These filters reduced the number of probe sets in each network to 5549 (CTX), 4050(CN), and 4029 (CB). After combining probes into single measures for each gene symbol and merging the data with the blood data sets, the CTX network contained 2640 genes, $\mathrm{CN}$ network contained 2063 genes and the $\mathrm{CB}$ network contained 2001 genes.

To determine whether a module found in a reference data set (e.g. human cortex) can also be found in a test data set (e.g. the Dutch blood data set), we used a powerful module preservation statistic approach implemented in the $\mathrm{R}$ software function modulePreservation [20] (described in Methods). This permutation test procedure evaluates whether module genes show significant evidence of network connectivity preservation in the test data. This module preservation test results in a statistic (referred to as preservation Z statistic or Zsummary statistic) for each module. The higher the preservation $Z$ statistic is for a given brain module, the stronger the evidence that the brain module is preserved in a given blood data set. Under the null hypothesis of no module preservation, the preservation $\mathrm{Z}$ statistic follows an approximately standard normal distribution. Comprehensive simulation studies led to the following thresholds: a module shows no evidence of preservation if its $\mathrm{Z}$ statistic is smaller than 2; a Z statistic larger than 5 (or 10) indicates moderate (strong) module preservation.

We started out by evaluating the preservation of CTX modules in the Dutch and SAFHS blood data sets. The horizontal barplots in Figure 1a show that the preservation Z statistics of the blue, yellow, and green CTX modules were above the threshold of 10 in both blood data sets, i.e. these modules showed strong evidence of preservation. Similarly, Figure 1b presents the module preservation results for the $\mathrm{CN}$ modules identified by Oldham et al. (2008). Only the yellow CN module was strongly preserved in both blood data sets. Figure 1c shows that only the blue $\mathrm{CB}$ module was strongly preserved in both blood data sets. In total, we find that five brain modules were strongly preserved in human blood. More details and numeric values are presented in Additional file 4 .

The preserved modules tended to be relatively large: Out of 2640 CTX network genes (from merging the CTX data with the blood data), 690 were part of the blue module, 421 were part of the green module and 658 were part of the yellow module. The preserved (yellow) $\mathrm{CN}$ module contained 254 genes out of $2063 \mathrm{CN}$ network genes. The preserved CB (blue) module contained 819 out of 2001
CB network genes. Thus, $67 \%$ of genes in the cortex network, $12 \%$ of genes in the caudate nucleus network, and $41 \%$ of genes in the cerebellum network were part of a preserved module.

One can also visualize the evidence of module preservation by clustering the genes in the blood data sets. Since the brain modules were defined as branches of a hierarchical clustering tree (dendrogram), we used the identical approach to define modules in the blood gene expression data. Additional file 5 shows dendrograms of the blood gene expression data. As described in the Methods section, blood modules were defined as branches of the dendrogram [20,21]. The first colorband underneath each dendrogram encodes blood module colors. The remaining color bands indicate module membership in brain modules. Visual inspection of these dendrograms revealed that genes from the preserved modules (based on the permutation test) tended to cluster together in the blood data. The fact that some colors were not contiguous shows that the preservation is not perfect. Below, we define measures of module membership to identify the subsets of genes inside each of the five preserved modules that showed the strongest evidence of preservation.

\section{Relationships among preserved modules in blood}

While the brain modules were clearly distinct in the brain data sets, their preserved counterparts no longer appeared distinct in the blood data sets. To explore the relationships among preserved modules in blood, we summarized module expression profiles by forming the first principal component, which is referred to as the module eigengene (ME) [21,24,25]. For example, ME (blue/CTX) denotes the module eigengene of the blue cortex module. The ME can be considered a weighted average of the gene expression profiles in a module. If the ME of one module is highly correlated with that of another module in the blood data, then the genes inside the two modules have similar blood expression patterns, i.e. the two modules cannot be distinguished.

For the Dutch data set and the SAFHS data set, Figure 2 shows that ME(blue/CTX), ME(blue/CB), ME(yellow/ $\mathrm{CTX}$ ), and $\mathrm{ME}$ (yellow/CN) had highly significant positive correlations $\left(r>=0.95, p<=10^{-40}\right)$ with each other, but highly significant negative correlations $(r<=-0.95, p<=$ $10^{-40}$ ) with $\mathrm{ME}($ green/CTX). This result indicates that the five preserved brain modules can hardly be distinguished in an unsigned gene co-expression network in blood, as they coalesce into one large preserved module. It is natural to ask whether these five modules were distinct in the original brain data sets. Additional file 6 shows that the three preserved CTX modules (blue/CTX, green/CTX, and yellow/CTX) were only moderately correlated in the CTX data: the correlation between ME(blue/CTX) and 


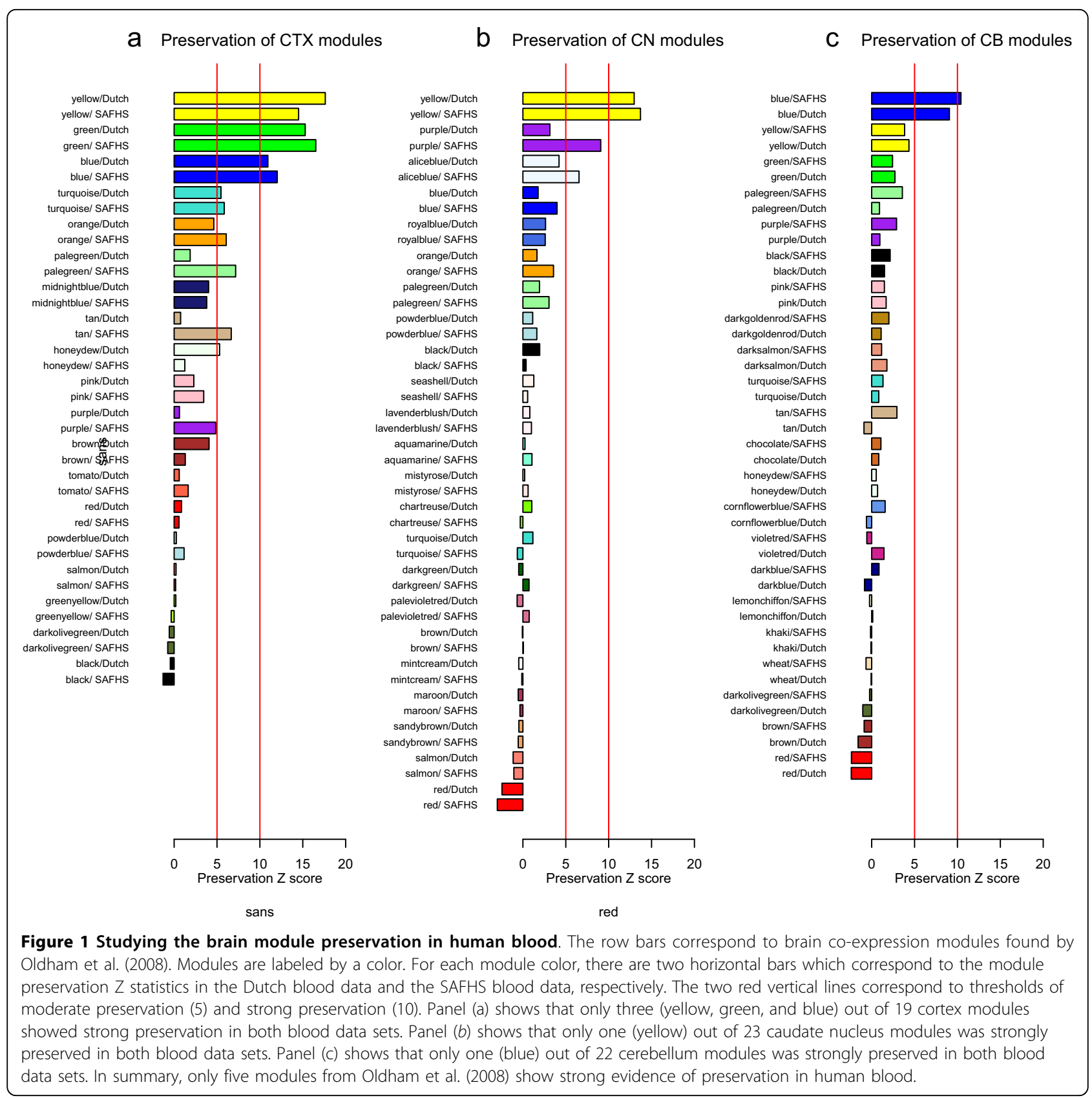

ME(yellow/CTX) was 0.52; the correlation between ME (blue/CTX) and ME(green/CTX) was -0.09; the correlation between ME(yellow/CTX) and ME(green/CTX) was -0.69 . The brain data did not allow us to correlate MEs of different brain regions, since the data consisted of samples from different individuals.

\section{Preservation of module membership between brain and blood}

We defined a measure of module membership (MM) by correlating the ME with each gene expression profile [26]. For example, MMblue $_{i}=\operatorname{cor}\left(x_{i}\right.$, MEblue $)$ measures how correlated the expression profile of the $\mathrm{i}$-th gene is with the blue ME. If $M_{M M b l u e}$ is close to 0 , the $i$-th gene is not part of the blue module. But if $\mathrm{MMblue}_{\mathrm{i}}$ is close to 1 (or -1 ), it is highly positively (or negatively) correlated with the blue module genes. The module membership measure is highly related to intramodular connectivity [26]; thus, a gene with high absolute value MMblue $_{i}$ turns out to be a highly connected hub gene inside the blue module.

For each of the five preserved modules, we defined module membership measures in the respective brain data set and the two blood data sets (Additional files 7, 


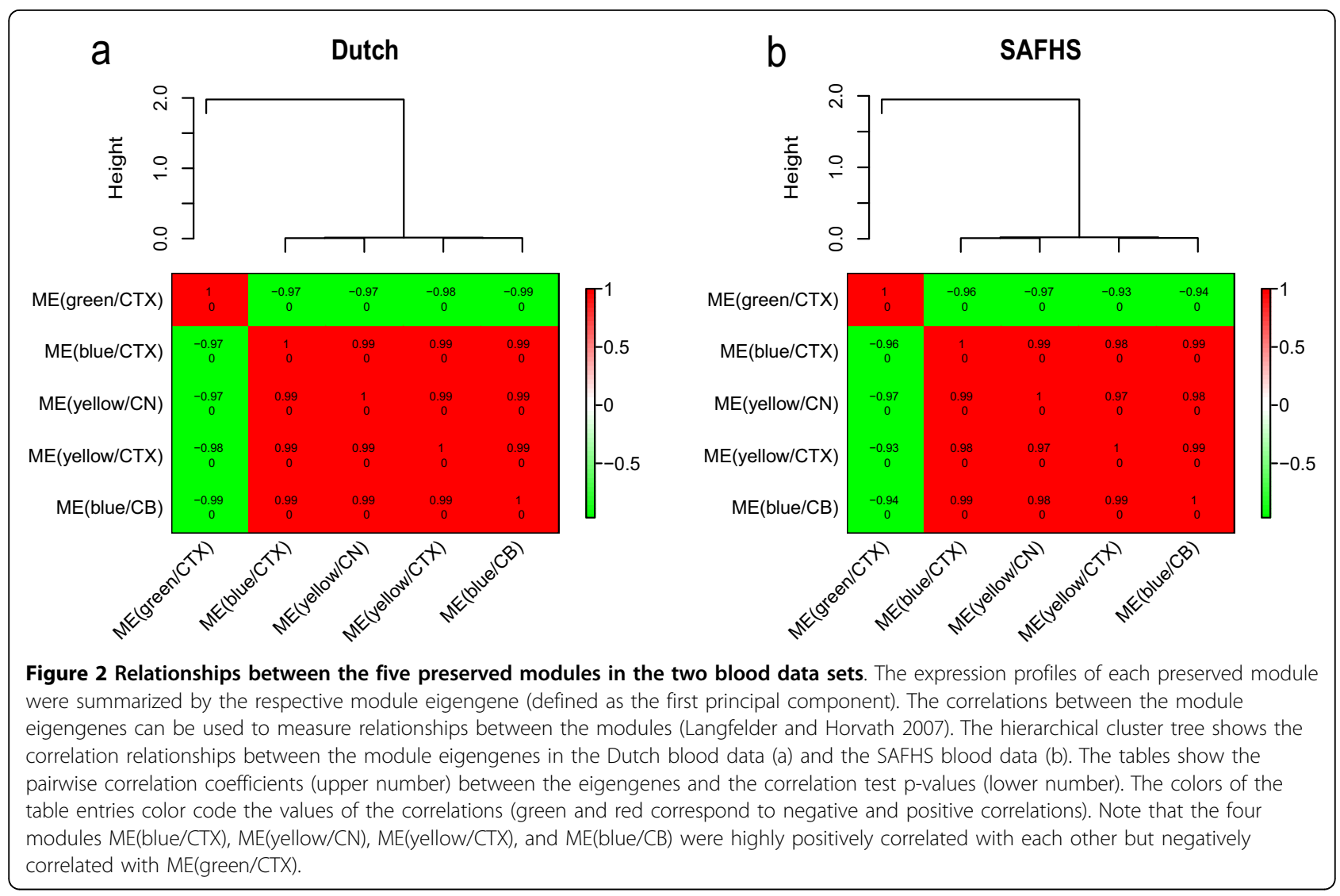

8 and 9). Figure 3 shows that MM measures were highly correlated between the two blood data sets, indicating that the MM measure can be robustly defined in blood.

The extremely significant correlation test p-values in the scatterplots reflect the large sample size, i.e. numbers of genes. It may be more meaningful to consider the correlation coefficient value, e.g. a correlation value of 0.76 indicates a strong (but not perfect) linear relationship. We combined the MM measures for the Dutch and SAFHS data to arrive at a summary measure for human blood, which was referred to as "Blood MM measure". Additional file 10 reports the correlations between the summary blood MM measure and the two individual blood MM measures.

Figure 4 shows that MM values for the three preserved CTX modules (yellow/CTX, green/CTX, and blue/CTX) were highly correlated in the blood data sets, which reflects what we already know from our eigengene-based analysis (Figure 2): these modules are indistinguishable in blood. Specifically, MM of yellow/CTX was positively correlated with MM of blue/CTX $(|r|=1$, $\mathrm{p}<10^{-200}$, Figure $\left.4 \mathrm{a}\right)$, while MM of green/CTX was negatively correlated with MM of both yellow/CTX and blue/CTX (Figure 4b-c). Given the exceptionally high correlations between the individual MM measures, it made sense to combine them by forming a weighted average, which flipped the sign of the negatively related green CTX module. We refer to the weighted average MM (across the modules) as the "combined MM measure". Additional file 11 shows that the combined MM value was highly correlated with the original MM value from the three modules.

Although the three modules were distinct in the cortex data, their MM measures also showed high correlations in cortex (Figure 4d-f), which allowed us to define a combined MM measure for the CTX data. The combined cortex MM measure was significantly correlated $\left(r=0.69, p<10^{-200}\right.$, Figure 5a) with the combined blood MM measure. At the same time, the $\mathrm{CN}$ MM measure and the CB MM measure also showed significant correlations with the blood MM measure $(r=0.45$, $p<2.9 \times 10^{-107}$, Figure 5b; $r=0.28, p<7.6 \times 10^{-38}$, Figure $5 \mathrm{c}$ ). These results support the original finding that the five co-expression modules (blue/CTX, green/ CTX, yellow/CTX, yellow/CN and blue/CB) exhibit significant preservation in blood.

\section{Definition of preserved intramodular hub genes}

We refer to genes with high module membership in a preserved module as a preserved intramodular hub 


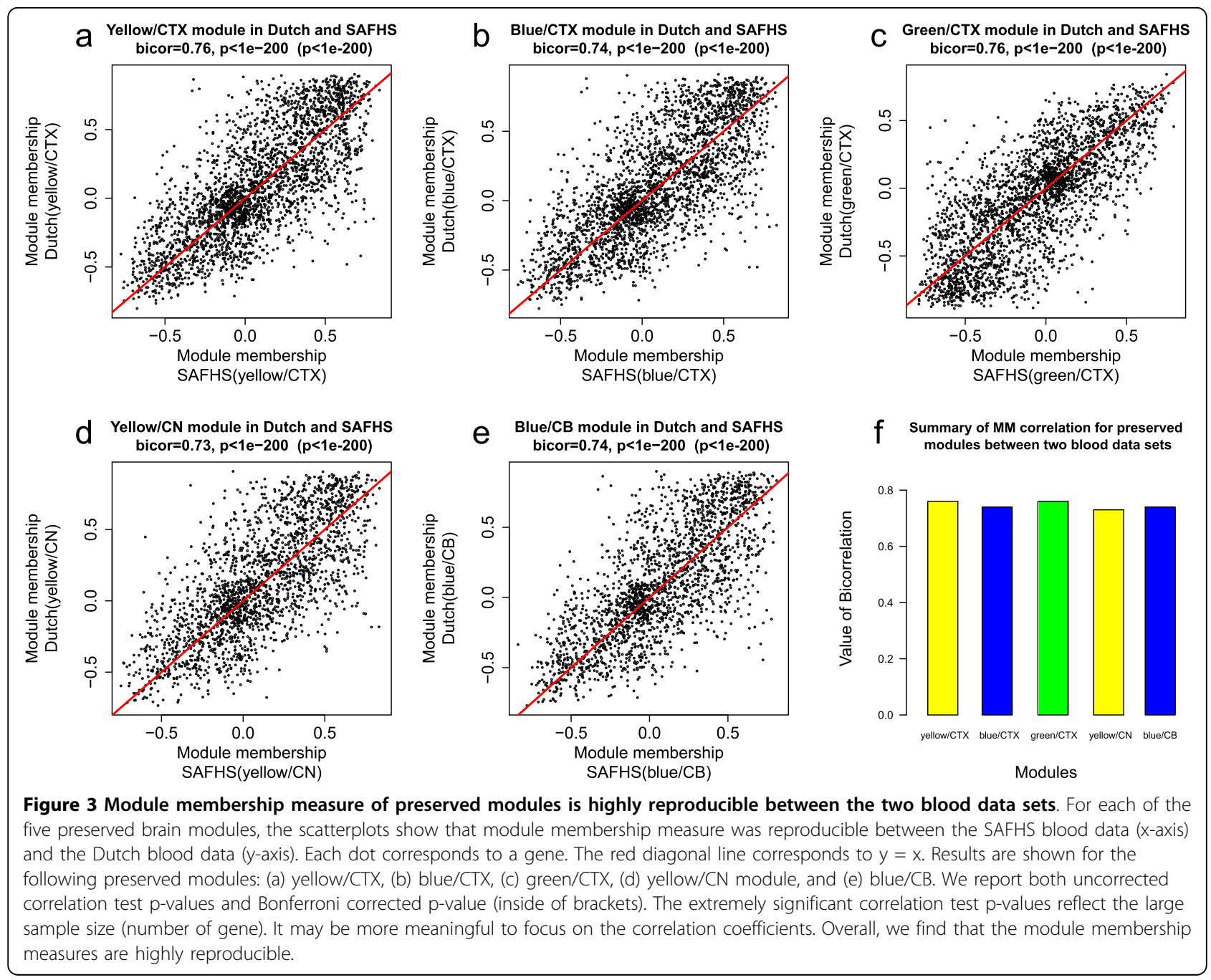

gene. Preserved hub genes show highly significant evidence of being centrally located inside a preserved module. Specifically, we defined preserved CTX module hub genes as having consistently high positive or negative combined MM in both cortex and blood. Toward this end, we thresholded the combined MM measures in both blood and cortex at a value of +0.35 and -0.35 (corresponding to a correlation test $p$-value $<5 \times 10^{-13}$ in blood). These thresholds resulted in 357 preserved CTX hub genes, which are colored in red in Figure 5a. For the preserved yellow $\mathrm{CN}$ module and preserved blue $\mathrm{CB}$ module, we found 305 preserved $\mathrm{CN}$ hub genes (colored yellow in Figure 5b) and 277 preserved CB hub genes (colored blue in Figure 5c) using the same threshold.

In summary, only 357 genes (13.5\%) from the CTX network, 305 genes (14.8\%) from the $\mathrm{CN}$ network and 277 genes $(13.8 \%)$ from the CB network are preserved intramodular hub genes. These preserved intramodular hub genes exhibited the following overlap: the sets of preserved CTX (357) genes and preserved CN (305) genes shared 123 genes (Fisher's exact $p$-value $<2.2 \times$ $10^{-16}$ ). The sets of preserved CTX (357) genes and preserved CB (277) genes shared 109 genes (Fisher's exact $p$-value $<2.2 \times 10^{-16}$ ). The sets of preserved $\mathrm{CN}$ (305) genes and preserved CB (277) genes shared 64 genes (Fisher's exact $p$-value $<1.8 \times 10^{-15}$ ). All three sets of preserved intramodular hub genes shared 36 genes. The names of these preserved hub genes and their MM values can be found in Additional file 12. The biological role of the 36 genes is discussed below.

The union of the three sets of preserved intramodular hub genes contains 678 genes. A functional enrichment analysis of the 678 genes reveals that some of these preserved hub genes play a role in infectious disease and infection mechanism $\left(p=8.6 \times 10^{-10}\right)$, post-translational modification $\left(p=2.4 \times 10^{-8}\right)$, and RNA post-transcriptional modification $\left(p=2.9 \times 10^{-8}\right)$ (Figure 6). A more 




detailed functional enrichment analysis for each set of preserved CTX, CN, and CB module genes can be found in Additional file 13.

\section{Preserved intramodular hub genes have more heritable expression levels}

In the original publication of the SAFHS data, the authors calculated the heritability of each gene expression level and created a heritability table [22]. The gene expression heritability measures the proportion of expression trait variance attributable to genetic variance. These data allowed us to test whether preserved intramodular hub genes have more highly heritable expression levels than non-preserved intramodular hub genes. The red, yellow and blue bars in Figure 5d-f show the mean heritability (y-axis) for the preserved CTX, CN and $\mathrm{CB}$ hub genes, respectively. To facilitate a comparison, we also report the mean heritability for all genes in heritability table (from Goring et al. 2008, grey bars) and for all genes in the merged blood and brain data set (black bars).

Figure 5d shows that that the preserved CTX hub genes ( $\mathrm{n}=357$, red bar) have a significantly (analysis of variance test $p=10^{-108}$ ) higher mean heritability (32\%) than all genes in heritability table $(\mathrm{n}=18525$, mean heritability: $23 \%$ ) and all genes in the CTX network ( $\mathrm{n}=$ 2640, mean heritability: 29\%). Analogous results were observed for the $\mathrm{CN}$ data set $\left(p=8.7 \times 10^{-92}\right.$, Figure 5e) and the CB data set $\left(p=8.1 \times 10^{-93}\right.$, Figure $\left.5 \mathrm{f}\right)$. These differences in heritability did not reflect differences in mean expression levels, as can be seen from Figure $5 \mathrm{~g}$-i, which report mean blood expression values (y-axis) across the different groups of genes. While preserved intramodular hub genes and brain network genes had significantly higher mean expression values than all genes in the heritability table (grey bar), preserved 


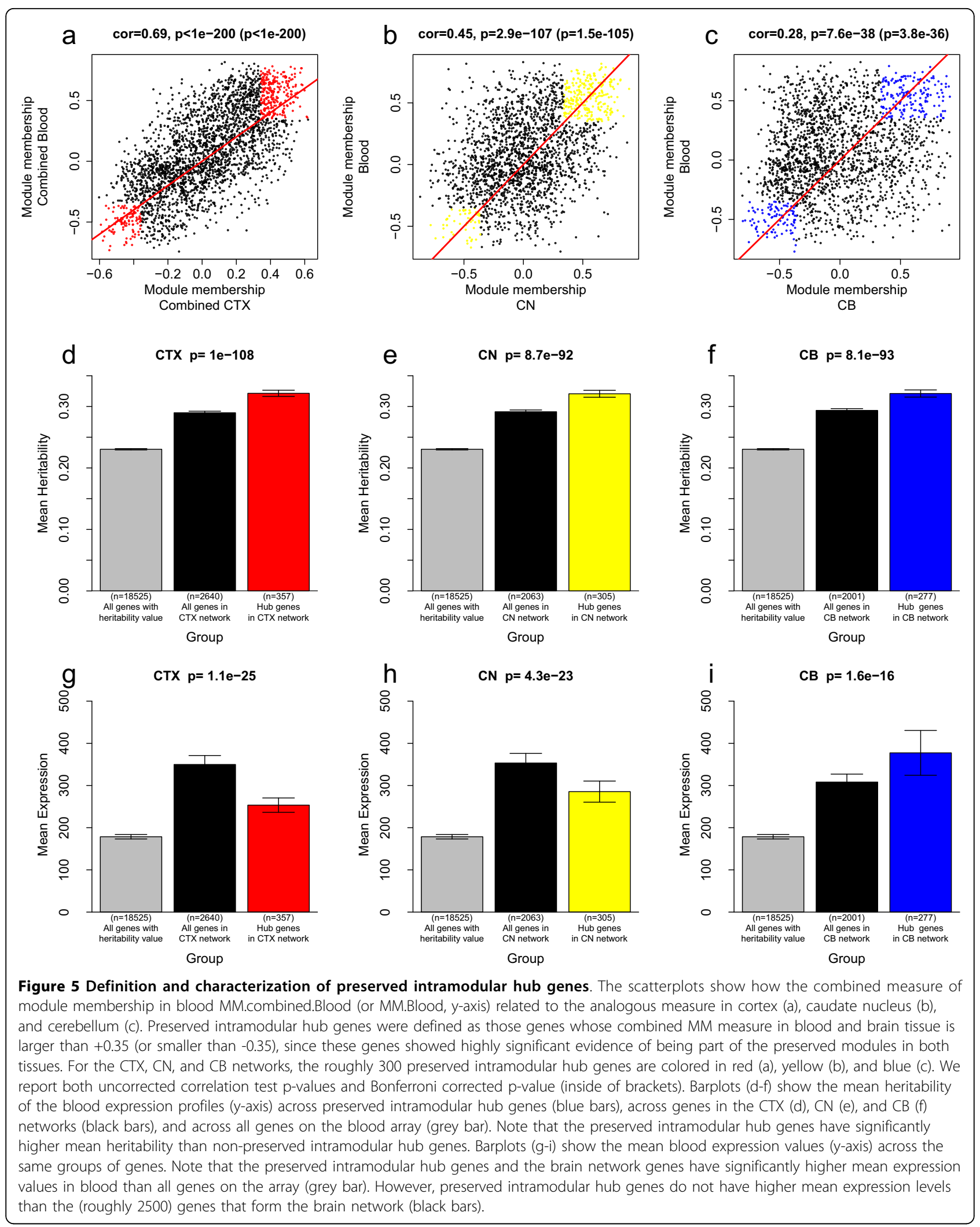




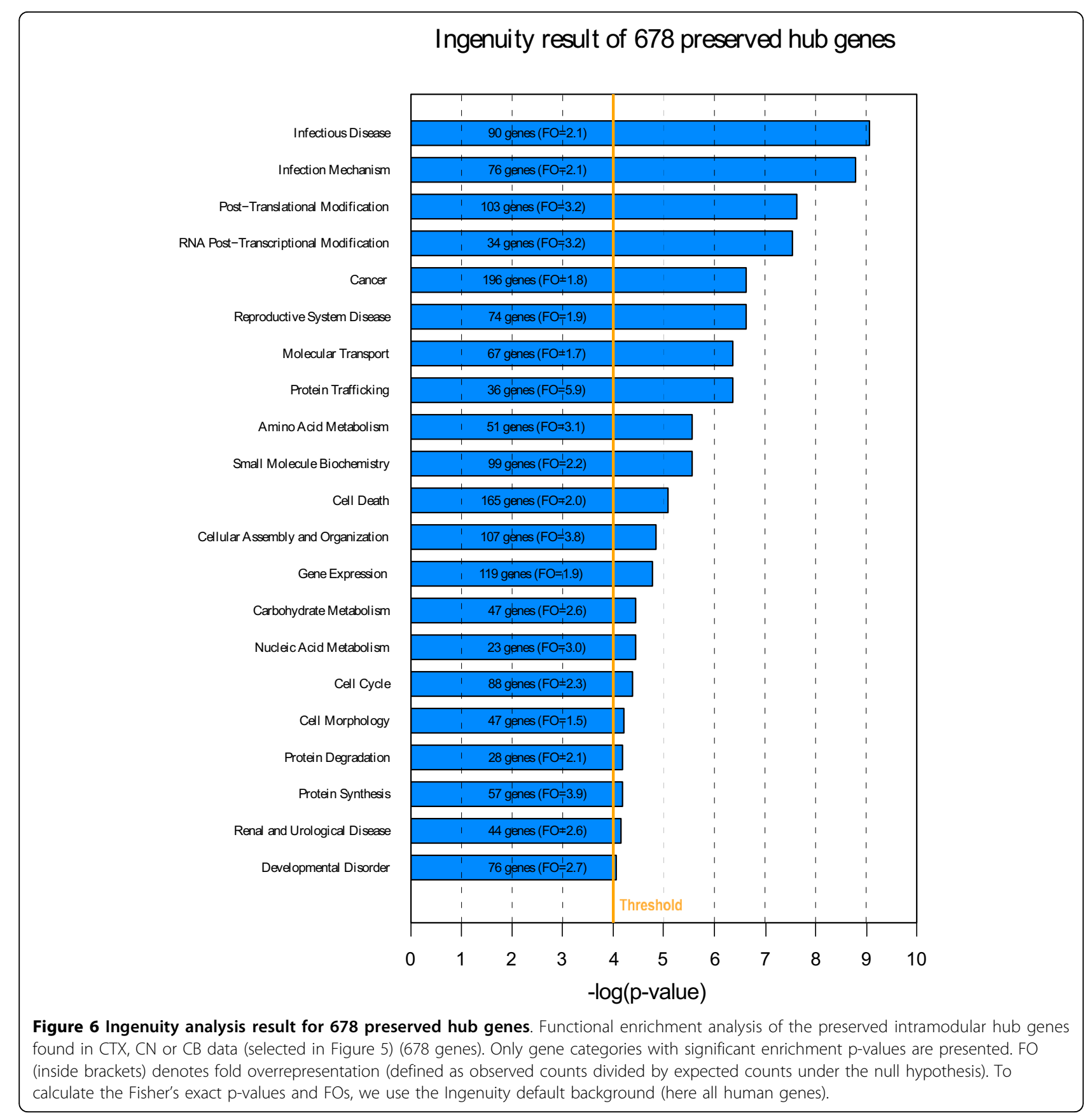

intramodular hub genes did not have higher mean expression levels than brain network genes (black bars).

\section{Relationships between preserved modules and cluster of differentiation genes}

We also investigated the relationships between the preserved modules and a special class of cell surface markers: cluster of differentiation $(C D)$ genes, which are routinely used to characterize blood cell types. If a module is enriched with cell type-specific genes, then its module eigengene should have a strong correlation with the expression values of $C D$ genes that are specific to that cell type. A high positive correlation would therefore suggest that a particular cell type might be related to the module. We found that the MEs of the five preserved modules had highly significant $\left(p<10^{-40}\right)$ positive correlations with the following $C D$ genes: $C D 58$, CD47, CD48, CD53 and CD164. Statistical details for the individual modules are presented in Additional file 14 . 
In the following, we briefly describe what is known about the products of these $\mathrm{CD}$ genes while Additional file 15 presents more detailed gene information (adapted from http://www.genecards.org and http://pathologyoutlines.com).

CD58 (present on Antigen Presenting Cells) is known to be a ligand of the $\mathrm{T}$ lymphocyte $\mathrm{CD} 2$ protein, and functions in adhesion and activation of $\mathrm{T}$ lymphocytes.

CD47 (present on leukocyte, neuroblast, glial cell and other cells) is a membrane protein, which is involved in the increase in intracellular calcium concentration that occurs upon cell adhesion to extracellular matrix.

CD48 (present on lymphocyte and other cells) is an activation-associated cell surface glycoprotein, and involved in facilitating interaction between activated lymphocytes.

CD53 (present on leukocyte, glial cell and other cells) is cell surface glycoprotein and involved in the regulation of cell development, activation, growth and motility.

CD164 (present on leukocyte, glial cell and other cells) is a type I integral transmembrane sialomucin that functions as an adhesion receptor. It is involved in hematopoiesis, migration of umbilical cord blood, prostate cancer metastasis, infiltration of bone marrow, myogenesis and myoblast migration.

\section{Module preservation between different brain regions}

As mentioned in the introduction, many brain modules were found to be highly preserved across the three brain regions, which is why they received the same color label. Here we use a more powerful approach for measuring module preservation (based on the modulePreservation $R$ function) than the one used in the original analysis by Oldham et al. Therefore, we use the modulePreservation function to re-analyze brain module preservation across brain regions. For example, we evaluate which CTX brain modules are preserved in the $\mathrm{CN}$ and $\mathrm{CB}$ data. Detailed results of this analysis can be found in Additional file 16. For CTX brain modules, we find that 11 out of 19 CTX module show at least moderate evidence of preservation (Preservation $\mathrm{Z}$ statistic $>5$ ) in both $\mathrm{CN}$ and $\mathrm{CB}$ data. For $\mathrm{CN}$ brain modules, we find that 12 out of $23 \mathrm{CN}$ modules also show at least moderate evidence of preservation (Preservation $\mathrm{Z}$ statistic $>5$ ) in both CTX and CB data. For CB brain modules, we find that 12 out of $22 \mathrm{CB}$ modules show at least moderate evidence of preservation (Preservation $\mathrm{Z}$ statistic $>5$ ) in both $\mathrm{CTX}$ and $\mathrm{CN}$ data. In summary, $55 \%$ modules showed preservation cross the different brain regions. These results are congruent with those presented in the original analysis by Oldham et al. It is particularly interesting to study which of our 5 preserved blood/brain modules are preserved in other brain regions.
For the 3 preserved CTX/blood modules (blue, green and yellow), we find that all 3 of them showed very high evidence of preservation in both $\mathrm{CN}$ (Preservation $\mathrm{Z}$ statistic $>=16.6$ ) and $\mathrm{CB}$ data (Preservation $\mathrm{Z}$ statistic $>=8.7$ ).

For the preserved (yellow) CN/blood module, we find very high evidence of preservation in CTX data (Preservation $\mathrm{Z}$ statistic $=19.1$ ), but only moderate/weak evidence preservation in $\mathrm{CB}$ data (Preservation $\mathrm{Z}$ statistic $=3.8$ ).

For the preserved (blue) $\mathrm{CB} /$ blood module, we find very high evidence of preservation in both $\mathrm{CN}$ (Preservation $\mathrm{Z}$ statistic $=20.8$ ) and $\mathrm{CB}$ data (Preservation $\mathrm{Z}$ statistic $>16.0$ ). Further, details can be found in Additional File 16.

Overall, we find strong evidence that the preserved brain/blood modules are also preserved in multiple brain regions.

\section{Discussion}

Few studies are able to access human neural tissue for studying diseases [27]. Given the difficulty of procuring human brain tissue versus the relative ease of measuring blood expression levels, a question of great practical importance is to determine to what extent blood is a reasonable surrogate for brain in gene expression studies. Here we relate highly reproducible brain expression data from a recent meta-analysis of human brain data sets to two large blood data sets. Overall, we find that mean expression levels are weakly preserved between three brain regions and blood ( $r$ range $[0.24,0.32])$. Since gene expression profiles in human brain regions are organized into highly reproducible co-expression modules [12], it is important to determine which of these modules show evidence of preservation in blood. Only three out of 19 cortex modules, one out of 23 caudate nucleus modules and one out of 22 cerebellum modules show strong evidence of preservation. In blood, these five modules exhibit very similar expression patterns as can be seen from the very high absolute correlations $(|r|$ $>0.96$ ) between their respective eigengenes (Figure 2).

Although few modules were preserved, they tended to be relatively large. $67 \%$ of genes in the cortex network were part of one of the three preserved modules; $41 \%$ of genes in the cerebellum network and $12 \%$ of the caudate nucleus network genes were part of their respective preserved modules. Intramodular hub genes inside preserved modules are centrally located in both modules. The number of intramodular hubs depends on the threshold used for the module membership measures in brain and blood. 13.5\% (357) of genes in the cortex network, $14.8 \%$ (305) of genes in the caudate nucleus network, and $13.8 \%$ (277) of genes in the cerebellum network were defined as preserved intramodular hub genes. Using our posted data and R software code, the 
reader can change the thresholds used for defining these hub genes. Our biological characterization of preserved intramodular hub genes is highly robust with respect to the chosen threshold values.

In mice, mean expression levels of heritable genes have been found to be highly correlated between mouse hippocampus and spleen [28]. We do not find that heritable genes exhibit highly correlated mean expression levels between brain and blood (Additional file 17). However, we find that the preserved intramodular hub genes tend to be more heritable (Figure 5).

The preserved CTX blue, green, and yellow modules were found to be enriched with neuronal markers, glutamatergic synapse genes, and metabolism-related genes, respectively. The preserved $\mathrm{CN}$ yellow module was also found to be enriched with metabolism-related genes, while the preserved CB blue module was enriched with neuronal markers and genes encoding synaptic proteins [12]. In blood, studying the enrichment with regard to brain cell type markers is not meaningful. However, one can classify blood cell types using human clusters of differentiation (CD) genes. Interestingly, the following CD molecules consistently have significant positive correlation with genes inside the preserved modules: CD58, CD47, CD48, CD53 and CD164.

A functional enrichment analysis of brain module preservation reveals basic functional pathways preserved between the two tissues. Figure 6 shows that these preserved intramodular hub genes are significantly enriched for genes that play a role in infectious disease and infection mechanism, post-translational modification and RNA post-transcriptional modification. Other categories include Cell Death, Energy Production, Nucleic Acid Metabolism, Molecular Transport and Protein Trafficking (Figure 6). The 36 intramodular hub genes that were preserved in all three sets exhibit several common functional themes. First, nearly $20 \%$ of these genes, including $A S F 1 A, A T F 2$, DR1, HCFC1R1, HMGN4, MBD3, and RAD21, are known to play roles in modifying chromatin structure. Some of these modifications have been shown to induce transcription (e.g. ATF2, DR1, HMGN4), while others produce repressive effects (e.g. $M B D 3$ ). A number of other genes in the group of 36 encode signalling proteins that are thought to play roles in a wide variety of cellular processes, including ARPP-19, CSNK1G3, MAP4K5, PPP1CB, and $Y W H A Q$. A third category of genes relates to protein trafficking and includes $R A B 1 A, S N X 2, S N X 3$, while a fourth category consists of genes involved in mitochondrial function, including DLAT, SUCLA2, and YME1L1. Some of the proteins encoded by these 36 genes may physically interact, such as ATP6AP2, which associates with the transmembrane sector of vacuolar ATPases (proton pumps), and ATP6V1C1, which is a subunit of the vacuolar ATPase protein complex. Intriguingly, for a number of other genes in this group, biological functions remained to be elucidated (e.g. FAM3C, FLJ20254, LANCL1, PRNP, $R A B G G T B$, and $W R B$ ). We note that many of these 36 preserved intramodular hub genes are expressed ubiquitously. Therefore, it is possible, perhaps even probable, that these genes are also co-expressed in other tissue types beyond brain and blood. Their co-expression may therefore help serve to maintain differentiated cells in a particular state (e.g. chromatin modifying genes) in response to a particular environment (e.g. signalling genes), as well as enable other shared, basic cellular processes (e.g. protein trafficking, energy metabolism).

Our study has several strengths including the use of multiple large data sets, carefully validated brain coexpression modules from Oldham et al 2008, and a powerful statistical approach for evaluating module preservation.

But our study also has several limitations including the following. First, the brain expression data were measured using the Affymetrix platform, while the blood expression data were measured using the Illumina platform. Since platform differences bias our results towards the null hypothesis of no preservation, we can be confident about preservation, but less confident about lack of preservation. The weak correlations between mean expression profiles may reflect platform differences. A second limitation is that we studied the preservation of brain modules in blood (and not vice versa). Our goal was to determine the preservation of robustly defined and well annotated brain modules. Defining blood modules and studying their preservation in brain tissue is beyond the scope of this article. A third limitation is the relatively small set of genes considered for the co-expression module preservation study. Oldham et al. had applied stringent filtering criteria to construct the brain network, which greatly reduced the number of probes considered in that study. After combining probes by gene symbol and merging the brain and blood data, the co-expression module preservation study focused on 2604 CTX, 2001 $\mathrm{CB}$, and $2063 \mathrm{CN}$ network genes. We focused on this relatively small set of genes since their connectivity pattern in brain was found to be highly reproducible across array platforms and independent data sets (Oldham et al 2008). But we should point out that our study of mean expression preservation involved 8799 genes. A fourth limitation is that we only use correlation network methodology. Many alternative co-expression network methods have been proposed in the literature $[27,29,30]$. We focus on WGCNA since i) this method was used in Oldham et al $(2008)$, ii) it is highly robust $[19,21]$, and iii) it affords a geometric interpretation of network concepts $[26,31]$. An exploration of alternative procedures is beyond our scope but we encourage the reader to apply their method to our posted data. 


\section{Conclusions}

In summary, we find that transcriptome organization is poorly preserved between brain and blood and only a handful of large brain co-expression modules that exhibit strong overall evidence of preservation in blood. However, these modules are not preserved whole cloth. Instead, only certain aspects of these modules (i.e. subsets of genes appear to be involved in basic cellular processes, such as metabolism) exhibit strong preservation of gene co-expression relationships. The subset of preserved co-expression relationships characterized here may aid future efforts to identify blood biomarkers for neurological and neuropsychiatric diseases when brain tissue samples are unavailable.

\section{Methods}

\section{Weighted gene co-expression network analysis and preservation visualization}

The statistical analysis software (WGCNA R package) and $\mathrm{R}$ tutorials for constructing a weighted gene coexpression network can be found in [20]. The WGCNA package first calculates all pairwise Pearson correlations coefficients across all samples. In a weighted network, the resulting Pearson correlation matrix is transformed into a adjacency matrix $\left(\mathrm{a}_{\mathrm{ij}}=\left|\operatorname{cor}\left(\mathrm{x}_{\mathrm{i}}, \mathrm{x}_{\mathrm{j}}\right)\right|^{\beta}\right)$, which represent the pairwise connection strengths. Here we use an unsigned network which ignores the sign of the corrlation relationship since this approach was used in the orignal brain data analysis [12]. However, we mention that one can also construct signed weighted networks that keep track of the sign of the correlation [32]. The power $\beta$ facilitates a soft-thresholding approach that preserves the continuous nature of the co-expression relationships [19-21]. As a power we chose the default value of 6 . An advantage of weighted networks is that they are highly robust with regard to the choice of the soft threshold parameter value. As a network dissimilarity measure we used 1 - the topological overlap measure as input for average linkage hierarchical clustering. The topological overlap measure is a highly robust measure of interconnectedness [33,34]. We used the dynamic branch cutting method to define modules as branches of the hierarchical clustering tree [35]. Unassigned background genes, outside of each of the modules, were denoted with the color grey.

\section{Connectivity and module membership measures}

Whole network connectivity for a certain gene is defined as the sum of its connection strengths with all other genes in the network. Mathematically, it can be calculated easily as the sum of a given column in the adjacency matrix. Intramodular connectivity is defined as the sum of the connection strengths between a particular gene and all other genes in the same module. Module membership (MM), or eigengene-based connectivity (kME), is another measure of connectivity. It is defined as $M^{\mathrm{q}}{ }_{\mathrm{i}}=$ $\operatorname{cor}\left(x_{i}, M E^{q}\right)$, where $x_{i}$ is the expression profile of $i$-th gene and $\mathrm{ME}^{\mathrm{q}}$ is the eigengene of q-th module. The larger the absolute values of $\mathrm{MM}$, the greater the similarity between the $\mathrm{i}$-th gene and the q-th ME. If the absolute value of $M M$ is 0 , it means that this gene is not part of the module. Although the MM measure is highly correlated with intramodular connectivity [26], the MM measure is preferred since it can be easily extended to genes outside the original module, and the statistical significance ( $p$-value) of MM can be calculated for every gene with respect to every module.

\section{Correlation tests}

To measure the relationship between brain tissue connectivity and blood tissue connectivity (and for relating mean expressions), we used a robust estimator of the correlation (the biweight midcorrelation implemented in the WGCNA R package) to protect against outliers. Simulation studies show that the biweight midcorrelation is more robust than the Pearson correlation but often more powerful than the Spearman correlation.

\section{Correcting $p$-values for multiple comparison tests}

To protect against false positives due to multiple testing, we also report Bonferroni corrected p-values. The Bonferroni correction method is the most conservative approach for correcting for multiple comparisons. The corrected p-value is defined by the product of the uncorrected p-value times the number of tests. Since we carried out 50 correlation tests in this article, a Bonferroni corrected $\mathrm{p}$-value is defined by multiplying the uncorrected p-values by 50 .

\section{Module Preservation analysis}

Our module preservation analysis is based on the modulePreservation $\mathrm{R}$ function implemented in the WGCNA $R$ package. The modulePreservation function implements several powerful network based statistics for evaluating module preservation. These statistics are summarized into the composite preservation called Zsummary. For each module in the reference data (e.g. brain data) one observed a value Zsummary in the test data (e.g. a blood data set). An advantage of the preservation $\mathrm{Z}$ statistic is that it makes few assumptions regarding module definition and module properties. Traditional cross-tabulation based statistics are inferior for the purposes of our study. While cross-tabulation approaches are intuitive, they have several disadvantages. To begin with, they are only applicable if the module assignment in the test data results from applying a 
module detection procedure to the test data. Even when modules are defined using a module detection procedure, cross-tabulation based approaches face potential pitfalls. A module found in the reference data set will be deemed non-reproducible in the test data set if no matching module can be identified by the module detection approach in the test data set. Such non-preservation may be called the weak non-preservation: "the module cannot be found using the current parameter settings of the module detection procedure". On the other hand, here we are interested in establishing strong non-preservation: "the module cannot be found irrespective of the parameter settings of the module detection procedure". Strong non-preservation is difficult to establish using cross-tabulation approaches that rely on module assignment in the test data set. A second disadvantage of a cross-tabulation based approach is that it requires that for each reference module one finds a matching test module. This may be difficult when a reference module overlaps with several test modules or when the overlaps are small. A third disadvantage is that cross-tabulating module membership between two networks may miss that the fact that the patterns of connectivity between module nodes are highly preserved between the two networks. The correlation network based statistics implemented in the modulePreservation function do not require the module assignment in the test network but require the user to input gene expression data underlying a reference data set and a test data set.

\section{Functional Enrichment Analysis}

The Ingenuity Pathways Analysis (Ingenuity ${ }^{\circ}$ Systems, http://www.ingenuity.com) software was used to determine whether sets of genes (e.g. preserved intramodular hub genes) were significantly enriched with known gene ontologies. This software ranks the pathways by their Fisher exact test p-value of functional enrichment. We chose the default background gene list (here all human genes) for the analysis. Ingenuity only reports uncorrected $\mathrm{p}$-values. The gene lists published in our Additional files allow the reader to choose alternative backgrounds or software tools.

\section{Additional material}

Additional file 1: Description of pre-process of each data Since data used in this manuscript are collected from different publications, each data used different platforms and different pre-process methods. This word document provides the detailed information of platform and preprocess methods which were provided by corresponding publications.

Additional file 2: Scatterplot of mean expression levels between brain and blood. This pairwise scatterplots relates mean expression of 8799 expressed genes from the CTX, CN and CB brain data sets with corresponding genes from the Dutch and SAFHS blood data sets respectively. We report a robust estimate of the correlation coefficient (biweight midcorrelation, see method section). In each plot, uncorrected p-value (without brackets) is reported, as well as Bonferroni corrected $\mathrm{p}$-value (with brackets). The extremely significant uncorrected correlation test $p$-values in scatterplots reflect the large sample size, i.e. numbers of genes. It may be more meaningful to focus on the correlation coefficient.

Additional file 3: Scatterplot of connectivity between brain and blood. This pairwise scatterplots relates connectivity of 8799 expressed genes from the CTX, CN and CB brain data sets with corresponding genes from the Dutch and SAFHS blood data sets respectively. We report a robust estimate of the correlation coefficient (biweight midcorrelation, see the method section). We report both uncorrected and Bonferroni corrected p-values (inside the brackets).

Additional file 4: Evaluating the preservation of brain modules in blood. This table reports the numeric preservation Z statistic for each brain module in both Dutch and SAFHS blood sets. Blue/CTX, green/CTX, yellow/CTX, yellow/CN and blue/CB modules consistently show significant preservation in both blood data sets.

Additional file 5: Cluster dendrogram of the blood data with different module annotations. The cluster dendrogram show the module definition based on the blood data. The first color band underneath the dendrogram shows the module assignment in blood. The remaining color bands show module assignment (for the preserved modules) based on the different brain regions. Visual inspection of these dendrograms reveals that genes from the preserved brain modules tend to cluster together in the blood data, which confirms the results of the module preservation Z statistics.

Additional file 6: Table of pairwise correlations between preserved CTX modules. Each preserved CTX module (yellow/CTX, blue/CTX and green/CTX) is represented by its eigengene. High positive or negative correlations between eigengenes suggest that the modules are indistinguishable. Correlations between the yellow/CTX, blue/CTX and green/CTX are reported.

Additional file 7: Module membership for preserved CTX modules This table reports the membership value for three preserved CTX modules (yellow/CTX, blue/CTX and green/CTX) in the CTX, Dutch and SAFHS datasets. The module membership (KME) reports the correlation between a gene expression profile and the module eigengene.

Additional file 8: Module membership for preserved CN module. This table reports the membership value for the preserved $\mathrm{CN}$ module (yellow/CN) in the CN, Dutch and SAFHS datasets. The module membership (KME) reports the correlation between a gene expression profile and the module eigengene.

Additional file 9: Module membership for preserved CB module. This table reports the membership value for the preserved $C B$ module (blue/CB) in the CB, Dutch and SAFHS datasets. The module membership (KME) reports the correlation between a gene expression profile and the module eigengene.

Additional file 10: Pairwise correlations of combined blood module membership and two individual blood module memberships. This table reports the pairwise correlations between the summary blood MM measure and the two individual blood MM measures for each preserved module (yellow/CTX, blue/CTX, green/CTX, yellow/CN and blue/CB). The table shows that the summary MM measure adequately represents the individual measures.

Additional file 11: Pairwise correlations of combined CTX module membership and three individual CTX module memberships. This table reports the correlations between the combined CTX MM measure (summarized from three preserved CTX modules' module membership) and three individual CTX module memberships. The table shows that the combined CTX MM measure adequately represents the individual measures.

Additional file 12: Preserved hub genes list and their module membership values. This table report three lists of preserved hub genes selected in Figure 5. Their corresponding module membership values are also provided. 
Additional file 13: Results of an Ingenuity analysis for each preserved hub genes. The table shows the Ingenuity functional enrichment for three preserved hub gene lists from Additional file 12 (only categories with $\mathrm{p}$-value smaller than $1.0 \mathrm{E}-4$ are shown). It also provides the detailed sub categories, as well as the corresponding genes.

Additional file 14: Relationship between modules and cluster of differentiation genes. Table of correlation coefficients (and p-values) between cluster of differentiation genes and preserved module eigengene

Additional file 15: Annotation of cluster of differentiation genes that correlate with preserved modules. Detailed functional annotation of significantly correlation cluster of differentiation genes selected from Additional file 14.

\section{Additional file 16: Studying the brain modules preservation in} other brain regions. Here we report the results from applying the modulePreservation function to evaluate module preservation between brain regions. For example, we evaluate whether CTX brain modules are preserved in $\mathrm{CN}$ and $\mathrm{CB}$ regions. We also evaluate the preservation of $\mathrm{CN}$ modules (and $\mathrm{CB}$ ) modules in the other regions. The bars in the barplots correspond to the preservation Z statistic of the modules. Each bar is colored by the original module color from Oldham et al.

\section{Additional file 17: Scatterplot of mean expression levels and}

heritability. This figure provides the scatterplot of mean expression level of top heritable genes with their heritability value. Uncorrected correlation test $\mathrm{p}$-values and Bonferroni corrected $\mathrm{p}$-values are reported (inside brackets).

\section{Abbreviations}

WGCNA: weighted gene co-expression network analysis; CD: cluster of differentiation; ME: module eigengene; MM: module membership; KME: eigengene-based connectivity.

\section{Acknowledgements}

We acknowledge grant support from P50CA092131, U19A1063603-01, P01HL028481, DE019255-01, R01DK072206.

\section{Author details}

'Department of Human Genetics, David Geffen School of Medicine, University of California Los Angeles, Los Angeles, CA 90095, USA. ${ }^{2}$ Department of Biostatistics, David Geffen School of Medicine, University of California Los Angeles, Los Angeles, CA 90095, USA. ²Department of Neurology, The Eli and Edythe Broad Center of Regeneration Medicine and Stem Cell Research, University of California San Francisco, San Francisco, CA 94143, USA. ${ }^{4}$ Department of Neurology, Rudolf Magnus Institute of Neuroscience, University Medical Center Utrecht, Utrecht 3584 CX, the Netherlands. 5 UCLA Center for Neurobehavioral Genetics, Semel Institute of Neuroscience and Human Behavioral, School of Medicine, University of California Los Angeles, Los Angeles, CA 90095, USA.

\section{Authors' contributions}

CC performed the research; $\mathrm{CC}, \mathrm{SH}, \mathrm{TFF}, \mathrm{PL}, \mathrm{RL}$, and $\mathrm{MCO}$ helped analyze the data; CC and SH wrote the paper. RAO and LHB provided and guided the analysis of the Dutch blood data sets. SH designed the research. All authors read and approved the final manuscript.

\section{Competing interests}

The authors declare that they have no competing interests.

Received: 2 June 2010 Accepted: 20 October 2010

Published: 20 October 2010

\section{References}

1. Jasinska AJ, Service S, Choi OW, DeYoung J, Grujic O, Kong SY, Jorgensen MJ, Bailey J, Breidenthal S, Fairbanks LA, et al: Identification of brain transcriptional variation reproduced in peripheral blood: an approach for mapping brain expression traits. Hum Mol Genet 2009, 18(22):4415-4427.
2. Sullivan PF, Fan C, Perou CM: Evaluating the comparability of gene expression in blood and brain. Am J Med Genet B Neuropsychiatr Genet 2006, 141B(3):261-268

3. Gladkevich A, Kauffman HF, Korf J: Lymphocytes as a neural probe: potential for studying psychiatric disorders. Prog Neuropsychopharmacol Biol Psychiatry 2004, 28(3):559-576.

4. Glatt SJ, Everall IP, Kremen WS, Corbeil J, Sasik R, Khanlou N, Han M, Liew CC, Tsuang MT: Comparative gene expression analysis of blood and brain provides concurrent validation of SELENBP1 up-regulation in schizophrenia. Proc Natl Acad Sci USA 2005, 102(43):15533-15538.

5. Matigian NA, McCurdy RD, Féron Fo, Perry C, Smith H, Filippich C, McLean D, McGrath J, Mackay-Sim A, Mowry B, et al: Fibroblast and Lymphoblast Gene Expression Profiles in Schizophrenia: Are Non-Neural Cells Informative? PLOS ONE 2008, 3(6):e2412.

6. Tsuang MT, Nossova N, Yager T, Tsuang MM, Guo SC, Shyu KG, Glatt SJ, Liew CC: Assessing the validity of blood-based gene expression profiles for the classification of schizophrenia and bipolar disorder: a preliminary report. Am J Med Genet B Neuropsychiatr Genet 2005, 133B(1):1-5.

7. Saris CG, Horvath $S$, van Vught PW, van Es MA, Blauw HM, Fuller TF, Langfelder $\mathrm{P}$, DeYoung J, Wokke JH, Veldink JH, et al: Weighted gene coexpression network analysis of the peripheral blood from Amyotrophic Lateral Sclerosis patients. BMC Genomics 2009, 10:405.

8. Borovecki F, Lovrecic L, Zhou J, Jeong H, Then F, Rosas HD, Hersch SM, Hogarth P, Bouzou B, Jensen RV, et al: Genome-wide expression profiling of human blood reveals biomarkers for Huntington's disease. Proc Natl Acad Sci USA 2005, 102(31):11023-11028.

9. Maes OC, Xu S, Yu B, Chertkow HM, Wang E, Schipper HM: Transcriptional profiling of Alzheimer blood mononuclear cells by microarray. Neurobiol Aging 2007, 28(12):1795-1809.

10. Presson A, Sobel E, Papp J, Suarez C, Whistler T, Rajeevan M, Vernon S, Horvath S: Integrated Weighted Gene Co-expression Network Analysis with an Application to Chronic Fatigue Syndrome. BMC Systems Biology 2008, 2(1):95.

11. Oldham MC, Horvath S, Geschwind DH: Conservation and evolution of gene coexpression networks in human and chimpanzee brains. Proc Natl Acad Sci USA 2006, 103(47):17973-17978.

12. Oldham MC, Konopka G, Iwamoto K, Langfelder P, Kato T, Horvath S, Geschwind DH: Functional organization of the transcriptome in human brain. Nat Neurosci 2008, 11(11):1271-1282.

13. Miller JA, Oldham MC, Geschwind DH: A systems level analysis of transcriptional changes in Alzheimer's disease and normal aging. J Neurosci 2008, 28(6):1410-1420.

14. Calvano SE, Xiao W, Richards DR, Felciano RM, Baker HV, Cho RJ, Chen RO, Brownstein BH, Cobb JP, Tschoeke SK, et al: A network-based analysis of systemic inflammation in humans. Nature 2005, 437(7061):1032-1037.

15. Hodges A, Strand AD, Aragaki AK, Kuhn A, Sengstag T, Hughes G, Elliston LA, Hartog C, Goldstein DR, Thu D, et al: Regional and cellular gene expression changes in human Huntington's disease brain. Hum Mol Genet 2006, 15(6):965-977.

16. Iwamoto K, Bundo M, Kato T: Altered expression of mitochondria-related genes in postmortem brains of patients with bipolar disorder or schizophrenia, as revealed by large-scale DNA microarray analysis. Hum Mol Genet 2005, 14(2):241-253.

17. Ryan MM, Lockstone HE, Huffaker SJ, Wayland MT, Webster MJ, Bahn S: Gene expression analysis of bipolar disorder reveals downregulation of the ubiquitin cycle and alterations in synaptic genes. Mol Psychiatry 2006, 11(10):965-978.

18. van der Merwe PA, McNamee PN, Davies EA, Barclay AN, Davis SJ: Topology of the CD2-CD48 cell-adhesion molecule complex: implications for antigen recognition by T cells. Curr Biol 1995, 5(1):74-84

19. Horvath S, Zhang B, Carlson M, Lu KV, Zhu S, Felciano RM, Laurance MF, Zhao W, Qi S, Chen Z, et al: Analysis of oncogenic signaling networks in glioblastoma identifies ASPM as a molecular target. Proc Natl Acad Sci USA 2006, 103(46):17402-17407.

20. Langfelder $P$, Horvath S: WGCNA: an R package for weighted correlation network analysis. BMC Bioinformatics 2008, 9:559.

21. Zhang B, Horvath S: A general framework for weighted gene coexpression network analysis. Stat Appl Genet Mol Biol 2005, 4, Article17.

22. Goring HH, Curran JE, Johnson MP, Dyer TD, Charlesworth J, Cole SA, Jowett JB, Abraham LJ, Rainwater DL, Comuzzie AG, et al: Discovery of 
expression QTLs using large-scale transcriptional profiling in human lymphocytes. Nat Genet 2007, 39(10):1208-1216.

23. Johnson WE, Li C, Rabinovic A: Adjusting batch effects in microarray expression data using empirical Bayes methods. Biostatistics 2007, 8(1):118-127.

24. Ghazalpour A, Doss S, Zhang B, Plaisier C, Wang S, Schadt E, Drake TA, Lusis A, Horvath S: Integrating Genetic and Network Analysis to Characterize Genes Related to Mouse Weight. PLoS Genet 2006, 2(8):e130.

25. Langfelder $P$, Horvath $\mathrm{S}$ : Eigengene networks for studying the relationships between co-expression modules. BMC Syst Biol 2007, 1:54.

26. Horvath S, Dong J: Geometric interpretation of gene coexpression network analysis. PLoS Comput Biol 2008, 4(8):e1000117.

27. Torkamani A, Dean B, Schork NJ, Thomas EA: Coexpression network analysis of neural tissue reveals perturbations in developmental processes in schizophrenia. Genome Research 20(4):403-412.

28. Davies MN, Lawn S, Whatley S, Fernandes C, Williams RW, Schalkwyk LC: To what extent is blood a reasonable surrogate for brain in gene expression studies: estimation from mouse hippocampus and spleen. Frontiers in Neurogenomics 2009, 3(54).

29. Keller MP, Choi Y, Wang P, Davis DB, Rabaglia ME, Oler AT, Stapleton DS, Argmann C, Schueler KL, Edwards S, et al: A gene expression network model of type 2 diabetes links cell cycle regulation in islets with diabetes susceptibility. Genome Res 2008, 18(5):706-716.

30. Stuart JM, Segal E, Koller D, Kim SK: A gene-coexpression network for global discovery of conserved genetic modules. Science 2003, 302(5643):249-255.

31. Dong J, Horvath S: Understanding network concepts in modules. BMC Syst Biol 2007, 1:24.

32. Mason M, Fan G, Plath K, Zhou Q, Horvath S: Signed weighted gene coexpression network analysis of transcriptional regulation in murine embryonic stem cells. BMC Genomics 2009, 10(1):327.

33. Yip AM, Horvath S: Gene network interconnectedness and the generalized topological overlap measure. BMC Bioinformatics 2007, 8:22.

34. Li A, Horvath S: Network neighborhood analysis with the multi-node topological overlap measure. Bioinformatics 2007, 23(2):222-231.

35. Langfelder $\mathrm{P}$, Zhang $\mathrm{B}$, Horvath $\mathrm{S}$ : Defining clusters from a hierarchical cluster tree: the Dynamic Tree Cut package for R. Bioinformatics 2008, 24(5):719-720.

doi:10.1186/1471-2164-11-589

Cite this article as: Cai et al:: Is human blood a good surrogate for brain tissue in transcriptional studies? BMC Genomics 2010 11:589.

\section{Submit your next manuscript to BioMed Central and take full advantage of:}

- Convenient online submission

- Thorough peer review

- No space constraints or color figure charges

- Immediate publication on acceptance

- Inclusion in PubMed, CAS, Scopus and Google Scholar

- Research which is freely available for redistribution

Submit your manuscript at www.biomedcentral.com/submit
Biomed Central 